\title{
A photometric study of the recently discovered eclipsing binary V899 Herculis
}

\author{
S. Özdemir, O. Demircan, A. Erdem, C. Çiçek, İ. Bulut, E. Soydugan, and F. Soydugan \\ Çanakkale Onsekiz Mart University, Department of Physics, Çanakkale, Turkey
}

Received 10 September 2001 / Accepted 18 January 2002

\begin{abstract}
The radial velocity and $U B V$ light curves of V899 Her as found in the literature were studied for the first time to obtain the orbital parameters and geometry of the system. The solutions obtained by using the Wilson-Devinney code reveal an over-contact system with a fill-out factor of $23.7 \%$. This solution is only possible with a large amount of third light $\left(L_{3} \approx 0.68\right)$ which confirms the spectroscopic result obtained by Lu et al. (2001) who reported that the system of which V899 Her is a fainter component is triple, or even quadruple. The distance of the system found by using absolute parameters is comparable with the Hipparcos result.
\end{abstract}

Key words. binaries: eclipsing - binaries: close - stars: individual: V899 Herculis

\section{Introduction}

The system V899 Her (HD 149684, HIP 81191) is among the recent photometric discoveries of the Hipparcos project (ESA, 1997). The Hipparcos photometric observations of the system show a W UMa type light curve with an amplitude of 0.121 ranging from $7^{\mathrm{m}} \cdot 935$ to $8^{\mathrm{m}} 056$ and with two minima of equal depth. The mean orbital period and the epoch derived by Hipparcos from the best light curve fitting are 0.421173 and JD 2448500.136. The spectral type of the system is given as F8.

The first ground-based $U B V$ photometry of the system was carried out by Özdemir et al. (2001a); the light curves they obtained show evidence of the O'Connell effect (O'Connell 1951), having slightly lower maxima at the second quadrature. They have also produced a corrected ephemeris of V899 Her by fitting a linear function to the Hipparcos data and times of minima.

The system has been recently observed spectroscopically by Lu et al. (2001). They reported that the system is composed of three, or even four, components among which the V899 Her system (component B) is the faintest; it exhibits weak and broadened G-type lines in its spectrum. They have also derived the radial velocity curves of the weak component (V899 Her) by using the broadening function and classified it as G-type.

In order to obtain full modelling of the system, the first ground-based photoelectric light curves (Özdemir et al. 2001a) and the radial velocity curves (Lu et al. 2001) were analyzed simultaneously.

Send offprint requests to: S. Özdemir, e-mail: ozdemir@comu.edu.tr

\section{The observational data}

The new photometric $U B V$ data presented by Özdemir et al. (2001a) are used to analyze the system. The light curves are folded by using their new ephemeris:

HJD Min I $=2448500.13600+0$ d $42117220(4) \times \mathrm{E}$.

The observations were made on 3 nights during the 2000 observing season using the equipments and technique described in Özdemir et al. (2001b). Their differential magnitudes derived from check star minus comparison star differences have standard errors of $0 .{ }^{\mathrm{m}} 020,0^{\mathrm{m}} 016$, and $0{ }^{\mathrm{m}} 021$ in the $U, B$, and $V$ passbands, respectively. All light curves including those from Hipparcos exhibit slight evidence of the O'Connell effect by showing higher maxima at the primary quadrature.

The spectroscopic data are from Lu et al. (2001). Their spectra, taken by an $1.8 \mathrm{~m}$ telescope, have a medium spectral resolution of about $R=10000-15000$. As they reported, V899 Her is a spectroscopic triple system in which the contact binary is the fainter one (component B). The bright companion (component $\mathrm{A}$ ), classified as F5 V according to their spectra, also has a radial velocity variance since they have noticed a well-defined variation in its spectrum during the 250 days of data. Lu et al. (2001) succeeded in resolving the radial velocity curves of V899 Her from the spectra dominated by the F5 star by applying a broadening function (Rucinski 1999). Their spectroscopic solution of V899 Her gives $q=0.566(2), V_{\gamma}\left(\mathrm{km} \mathrm{s}^{-1}\right)=$ $-16.84 \pm 1.30$ and $f(M)=\left(M_{1}+M_{2}\right) \sin ^{3} i=(2.33 \pm$ $0.08) M_{\odot} \cdot$ 


\section{The solutions}

The $U B V$ light curves normalized to unity and the radial velocity curves of V899 Her have been used as input into the Wilson-Devinney (WD) code (Wilson 1992) to obtain a simultaneous solution. A total of 281, 285 and 279 data points were used for each $U B V$ filter, respectively, and a total of 53 data points of each radial velocity curve was considered in the solutions. Three different configurations were tested to obtain solutions: i) an over-contact mode (MOD 01) with some constrained parameters for the secondary which are the omega potential $\left(\Omega_{2}\right)$, temperature $\left(T_{2}\right)$, albedo $\left(A_{2}\right)$, gravity darkening $\left(g_{2}\right)$, and limb darkening coefficient $\left(x_{2}\right)$, ii) a semi-detached mode (MOD 04) which has the constraints that the omega potential of the primary $\left(\Omega_{1}\right)$ is fixed to the lobe-filling value, and that the temperature of the secondary $\left(T_{2}\right)$ is coupled to the temperature of the primary, and iii) an overcontact mode (MOD 03) the same as mode 1, except that the constraint on the temperature of the secondary $\left(T_{2}\right)$ is not applied. The differential corrections proposed by MOD 04 yield an over-contact configuration. The profiles of the light curves also offer some clues about Roche lobe filling components. It was therefore decided to apply only contact configurations. The solutions are given in Table 1 and expressed graphically in Fig. 1.

While calculating the solution, some parameters were set at reliably known values: $g_{1,2}=0.32$ (see Lucy 1967 for the gravity darkening exponents of convective atmospheres), $x_{1,2}(U, B, V)=0.847,0.789,0.684$ taken from Díaz-Cordovés et al. (1995), $F_{1,2}=1.0, A_{1,2}=1.0$, and $e=0.0$. The mass ratio $(q)$, the effective temperature of the primary component of V899 Her $\left(T_{1}\right)$, and the velocity of the center of mass $\left(V_{\gamma}\right)$ are set to the values of $\mathrm{Lu}$ et al.'s (2001) spectroscopic solution. The primary temperature $\left(T_{1}\right)$ was set to $5700 \mathrm{~K}$ on the basis of $\mathrm{Lu}$ et al.'s (2001) estimate that the fainter component (V899 Her B) is a G-type. A second reason why we have chosen the primary temperature as around Solar type is that this temperature is just about the limit at which both components of the system (viz. F5V + G) can be seen in the same spectrum. Other values, such as the distance between components $(a)$, orbital inclination $(i)$, surface temperature of the secondary $\left(T_{2}\right)$, dimensionless omega potentials $\left(\Omega_{1,2}\right)$, fractional luminosities $\left(L_{1,2}\right)$, and the contamination of the third light are adjustable during differential iterations.

Though the spectroscopy yields definite values for the mass ratio and the gamma (space) velocity of the system, these parameters have been allowed to change freely in MOD 01 by considering an input data set without radial velocity curves. This test showed that the results of $q$ and $V_{\gamma}$ do not differ from the solution given in Table 1, which confirms that the spectroscopic and photometric solutions are in good agreement.

The free parameters increased the amount of the third light through differential iterations up to the values listed in Table 1. The level of the third light confirms the
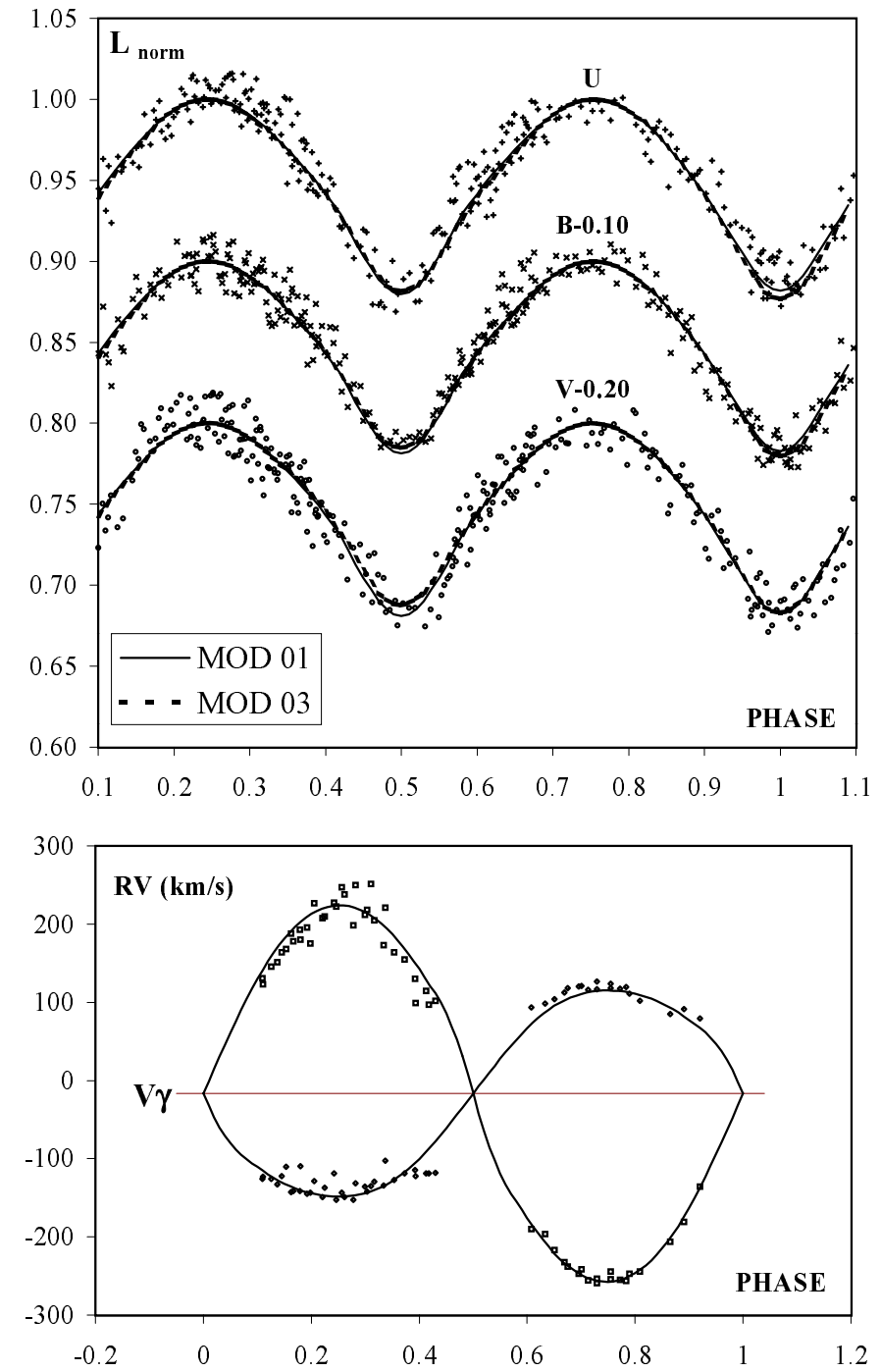

Fig. 1. The light curve (top panel) and radial velocity curve (bottom panel) solutions of the V899 Her system.

spectroscopic discovery of a third component by Lu et al. (2001). However, the photometric value in Table 1 is considerably larger than what is found from spectroscopy, viz. $L_{3} / L_{1,2}=1.5 \pm 0.1$. We shall return later to this inconsistency.

There is evidence of the O'Connell effect with slightly lower secondary quadrature. The difference between the maxima is estimated to be $0.015,0.003$, and 0.008 , in $U, B$, and $V$, respectively. These results indicate that the light levels in the secondary maxima are low in all passbands.

\subsection{The absolute parameters}

The simultaneous solutions of the photometric and radial velocity curves yield absolute parameters of V899 Her. The results are listed in Table 2. The calculations of the absolute parameters and their errors were based on the MOD 01 solution of Table 1 . The radii used in deriving absolute dimensions are the values for the geometric mean: $\left(r_{\text {pole }} \cdot r_{\text {back }} \cdot r_{\text {side }}\right)^{1 / 3}$ of the Roche geometry. 
Table 1. The simultaneous solutions of the light and radial velocity curves of V899 Her in MOD 01 and MOD 03 of the WD code. The parameters without errors are coupled to the other parameters or fixed. $L_{\text {Total }}$ equals $L_{1}+L_{2}+L_{3}$.

\begin{tabular}{|c|c|c|}
\hline Parameter & MOD 01 & MOD 03 \\
\hline$a\left(R_{\odot}\right)$ & $3.511 \pm 0.310$ & $3.511 \pm 0.318$ \\
\hline$e$ (eccentricity) & 0.0 & 0.0 \\
\hline$F_{1}=F_{2}$ & 1.0 & 1.0 \\
\hline$V_{\gamma}\left(\mathrm{km} \mathrm{s}^{-1}\right)$ & -16.8 & -16.8 \\
\hline$i\left(^{\circ}\right)$ & $68.720 \pm 0.159$ & $65.772 \pm 0.186$ \\
\hline$g_{1}=g_{2}$ & 0.32 & 0.32 \\
\hline$T_{1}(\mathrm{~K})$ & 5700 & 5700 \\
\hline$T_{2}(\mathrm{~K})$ & 5677 & $5550 \pm 3$ \\
\hline$A_{1}=A_{2}$ & 1.0 & 1.0 \\
\hline$\Omega_{1}$ & $2.922 \pm 0.005$ & $2.9407 \pm 0.004$ \\
\hline$\Omega_{2}$ & 2.9220 & 2.9407 \\
\hline$q\left(M_{2} / M_{1}\right)$ & 0.566 & 0.566 \\
\hline$r_{1}($ pole $)$ & $0.4168 \pm 0.0009$ & $0.4137 \pm 0.0003$ \\
\hline$r_{1}$ (side) & $0.4445 \pm 0.0012$ & $0.4404 \pm 0.0004$ \\
\hline$r_{1}$ (back) & $0.4788 \pm 0.0017$ & $0.4733 \pm 0.0006$ \\
\hline$r_{2}($ pole $)$ & $0.3229 \pm 0.0010$ & $0.3197 \pm 0.0005$ \\
\hline$r_{2}$ (side) & $0.3395 \pm 0.0012$ & $0.3355 \pm 0.0007$ \\
\hline$r_{2}$ (back) & $0.3809 \pm 0.0020$ & $0.3745 \pm 0.0012$ \\
\hline$L_{1}(U)\left(L_{1} / L_{\text {Total }}\right)$ & $0.199 \pm 0.028$ & $0.2455 \pm 0.034$ \\
\hline$L_{1}(B)\left(L_{1} / L_{\text {Total }}\right)$ & $0.204 \pm 0.023$ & $0.242 \pm 0.027$ \\
\hline$L_{1}(V)\left(L_{1} / L_{\text {Total }}\right)$ & $0.212 \pm 0.033$ & $0.242 \pm 0.038$ \\
\hline$L_{2}(U)\left(L_{2} / L_{\text {Total }}\right)$ & 0.117 & 0.123 \\
\hline$L_{2}(B)\left(L_{2} / L_{\text {Total }}\right)$ & 0.121 & 0.127 \\
\hline$L_{2}(V)\left(L_{2} / L_{\text {Total }}\right)$ & 0.125 & 0.129 \\
\hline$x_{1}(U, B, V)$ & $0.847,0.789,0.684$ & $0.847,0.789,0.684$ \\
\hline$x_{2}(U, B, V)$ & $0.847,0.789,0.684$ & $0.847,0.789,0.684$ \\
\hline$L_{3}(U)\left(L_{3} / L_{\text {Total }}\right)$ & $0.684 \pm 0.003$ & $0.631 \pm 0.004$ \\
\hline$L_{3}(B)\left(L_{3} / L_{\text {Total }}\right)$ & $0.675 \pm 0.003$ & $0.630 \pm 0.003$ \\
\hline$L_{3}(V)\left(L_{3} / L_{\text {Total }}\right)$ & $0.663 \pm 0.004$ & $0.629 \pm 0.005$ \\
\hline$\chi^{2}$ & 0.035 & 0.035 \\
\hline
\end{tabular}

Using absolute luminosities of $L_{1}, L_{2}, L_{3}$, and the distance modulus, we find a distance to the system of $\approx 104$ parsecs. This is reasonably consistent with the Hipparcos result which is $124 \pm 12$ parsecs. Considering the Hipparcos parallax and the absolute values of the eclipsing system, the distance between the component stars $(a)$ found to be $a=3.51 R_{\odot}$ confirms the WD results (see Table 1).

\section{Conclusions and remarks}

An analysis of new photometric and spectroscopic data of the close binary V899 Her discovered by Hipparcos revealed that the system is an over-contact binary and has a bright third component.
Table 2. The absolute dimensions of V899 Her according to the MOD 01 solution given in Table 1. $E(B-V)$ is assumed to be 0.0 in the calculation of $M_{\mathrm{bol}}$.

\begin{tabular}{|c|c|c|c|}
\hline Parameter & $\begin{array}{c}\text { Component B } \\
\text { Primary }\end{array}$ & $\begin{array}{c}\text { Component B } \\
\text { Secondary }\end{array}$ & Component $\mathrm{A}$ \\
\hline$M\left(M_{\odot}\right)$ & $2.10 \pm 0.15$ & $1.19 \pm 0.08$ & \\
\hline$R\left(R_{\odot}\right)$ & $1.57 \pm 0.14$ & $1.22 \pm 0.14$ & \\
\hline$T(K)$ & 5700 & 5677 & $6540^{*}$ \\
\hline$L\left(L_{\odot}\right)$ & $2.32 \pm 0.22$ & $1.40 \pm 0.17$ & $7.74 \pm 0.80$ \\
\hline$a\left(R_{\odot}\right)$ & $1.57 \pm 0.14$ & $1.22 \pm 0.14$ & \\
\hline$<\rho>(\operatorname{cgs})$ & $0.77 \pm 0.10$ & $0.93 \pm 0.15$ & \\
\hline $\log g(\operatorname{cgs})$ & $4.371 \pm 0.013$ & $4.342 \pm 0.018$ & \\
\hline$M_{\text {bol. }}\left({ }^{\mathrm{m}}\right)$ & $+3.84 \pm 0.09$ & $+4.38 \pm 0.12$ & $+2.53 \pm 0.10$ \\
\hline fill - out & $23 .(7) \%$ & & \\
\hline$d(\mathrm{pcs})$ & $124 \pm 12$ & & \\
\hline
\end{tabular}

* Adopted from the spectral type (F5 V).

Although the solutions resemble an A-subclass of W UMa type systems, some discrepancies with the Aclassification do exist, i.e.: i) V899 Her has a later spectral type (assumed G2-3, Solar type), ii) The system has a convective common envelope with a fill-out factor of $23.7 \%$ whereas the A-subclass has been identified with radiative atmospheres and relatively early type spectra by Binnendijk (1970, 1977). However, the remaining properties, such as transit primary minimum, degree of fill-out factor, the presence of a hotter, larger and much more massive primary component, and the total mass of the V899 Her system, are consistent with A-subclass (see e.g. Samec et al. 1996; Hilditch et al. 1988). Another nonnegligible probability is that the system may be in a minimum phase of magnetic activity, as the almost equal maxima levels indicate. When the magnetic activity rises, the O'Connell effect will increase and most probably the system will belong to the $\mathrm{W}$-subclass, due to the spotted primary component.

One can estimate the total mass of the eclipsing system as approximately $(2.9 \pm 0.1) M_{\odot}$ from the spectroscopic mass function of Lu et al. (2001) by using the photometric inclination $\left(i=68^{\circ} .7\right)$ from Table 1 . This spectroscopic mass of the eclipsing system is within an acceptable range (see Table 2). A comparison of the primary and secondary masses with the empirical mass vs. spectral type relation of Popper (1980) and with the theoretical stellar parameters derived by Straizy \& Kuriliene (1981) indicates that the components have to be evolved from the ZAMS. Figure 2 represents the Roche geometry of V899 Her system together with the surface outlines seen from the orbital plane, as given by the solution.

In a work of Van't Veer (1978), where the mass ratio function of W UMa type binaries is studied, his Fig. 1 showing orbital inclination vs. mass ratio for different amplitudes, is used for comparison with V899 Her: if the contribution of the third light is not included, the expected 


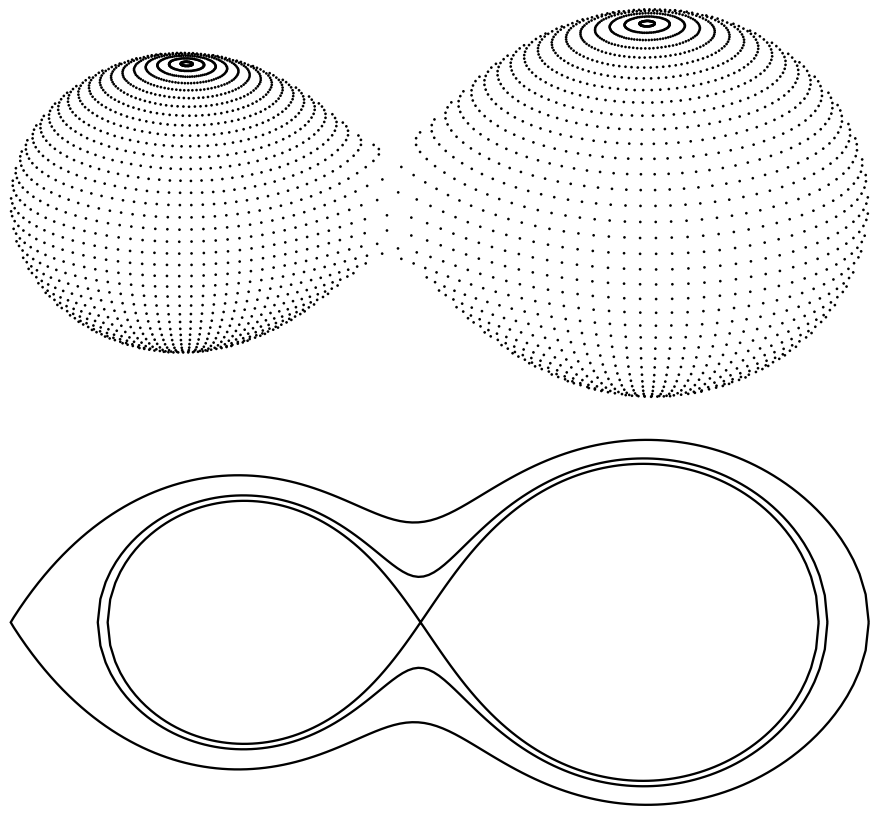

Fig. 2. The Roche geometry (top) and surface outlines (bottom) of V899 Her at phase 0.75.

amplitude (0.502) from the light curve of V899 Her matches an inclination of $\approx 73^{\circ}$ which is consistent with this simultaneous solution. Otherwise, if the observed light curves reduced by the third light are taken into account, the average amplitude $(0.137)$ corresponds to an inclination of $\approx 47^{\circ}$ which is outside the acceptable solution limits.

The light contamination by the third body, of around $68 \%$ of the total light, can not be ignored, as seen from Fig. 3. V899 Her is, therefore, a good example of eclipsing binaries which has evident third light contamination. However, all the available data of V899 Her from the spectroscopy and photometry do not permit us to obtain full orbital parameters of the third body.

High-dispersion spectroscopy to resolve the spectral lines, astrometry to determine the position, or the determination of long-term period changes to obtain the distance of the third body to the system will be helpful to discover the nature of the third, or perhaps fourth component of the V899 Her system. The system deserves to join the list of binary stars with possible additional components as a new member. A recent list of eclipsing binaries with evidence of additional components can be found in Demircan (2000).

The simultaneous solutions of the photometric and spectroscopic data reveal a fractional luminosity of the third light of $\left(L_{3} / L_{1,2}\right)=2.08 \pm 0.15$ (MOD 01) which exceeds by about 0.58 that of Lu et al.'s (2001) spectroscopic finding. The fact that using two different solution techniques, the spectral broadening function (Rucinski 1999) used in resolving radial velocity curves, and the data handling of the Wilson-Devinney code, may have caused such a disagreement.

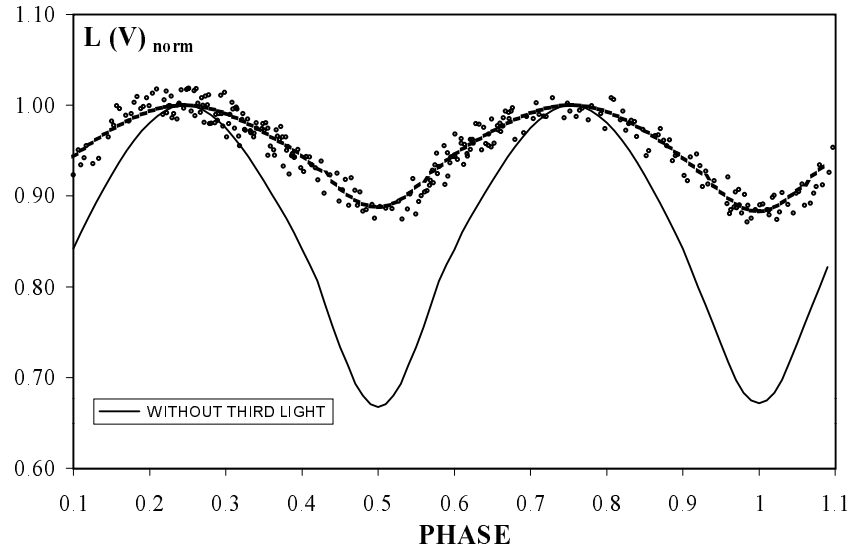

Fig. 3. A comparison between the light curves with and without third light. Notice how the third light reduces the amplitude of the light curve.

Some remarkable differences between the solution modes can be seen in Table 1 although mode 03 is the same as mode 01 except for the adjustable temperature of the secondary $\left(T_{2}\right)$. The mode 03 parameters, which can clearly be a solution for the V899 Her system, are only listed to give an idea about the results of different contact modes. However, mode 01 is preferable since the system currently seems to be an A-subclass of W UMa systems in which any remarkable temperature difference between the components is not believable.

Acknowledgements. This work was supported partly by the Scientific and Technical Research Council of Turkey (Tübitak), and the Research Fund of Çanakkale Onsekiz Mart University. The authors thank the referee for his helpful discussions and suggestions.

\section{References}

Binnendijk, L. 1970, Vistas Astr., 12, 217

Binnendijk, L. 1977, Vistas Astr., 21, 359

Demircan, O. 2000, in Variable Stars as Essential Astrophysical Tools, ed. C. İbanoğlu, NATO-ASI Ser. C, v. 544, 615

Díaz-Cordovés, J., Claret, A., \& Giménez, A. 1995, A\&AS, 110,329

ESA 1997, The Hipparcos \& Tycho Catalogues, ESA SP-1200

Hilditch, R. W., King, D. J., \& McFarlane, T. M. 1988, MNRAS, 231, 341

Lu, W., Rucinski, S. M., \& Ogloza, W. 2001, ApJ, 122, 402

Lucy, L. B. 1967, Z. Astrophys., 65, 89

O'Connell, D. J. K. 1951, Pub. Riverv. Coll. Obs., 2, 85

Özdemir, S., Demircan, O., Erdem, A., et al. 2001a, IBVS, 5034

Özdemir, S., Demircan, O., Erdem, A., et al. 2001b, IBVS, 5033

Popper, D. M. 1980, ARA\&A, 18, 115

Rucinski, S. 1999, in Precise Stellar Radial Velocities, ed. J. B. Hearnshaw, \& C. D. Scarfe, ASP Conf. Ser., 185, 82

Samec, R. G., Gray, J. D., \& Carrigan, B. J. 1996, The Observatory, 116, 75

Straizy, V., \& Kuriliene, G. 1981, Ap\&SS, 80, 353

Van't Veer, F. 1978, A\&A, 70, 91

Wilson, R. E. 1992, Documentation of Eclipsing Binary Computer Model, Revision of 1992: May, University of Florida 